\title{
Tumor Protein p53 and K-ras Gene Mutations in Peruvian Patients with Gallbladder Cancer
}

\author{
Tatiana Vidaurre ${ }^{1}$, Sandro Casavilca ${ }^{1}$, Paola Montenegro ${ }^{1}$, Henry Gomez ${ }^{1}$, \\ Mónica Calderón', Jeannie Navarro ${ }^{1}$, Jessica Aramburu', Ebert Poquioma ${ }^{1}$, \\ Yasuo Tsuchiya $^{2 *}$, Takao Asai ${ }^{2,3}$, Yoichi Ajioka ${ }^{4}$, Ayako Sato ${ }^{4}$, Toshikazu Ikoma ${ }^{2,5}$, \\ Kazutoshi Nakamura ${ }^{2}$
}

\begin{abstract}
Background: Recent studies have shown that genetic alterations are associated with the effect of patient geographic location on gallbladder cancer development. Peru has a high incidence of gallbladder cancer, but causative factors have not yet been identified. We examined the frequency of mutations in TP53 and K-ras genes in Peruvian patients with gallbladder cancer, and compared this with data from Bolivia, Hungary, Chile, and Japan, which have a high gallbladder cancer incidence. Methods: DNA was extracted from formalin-fixed paraffin-embedded gallbladder tissue sections of 30 gallbladder cancer patients ( 9 men and 21 women) obtained using microdissection. Mutations in exons 5 to 8 of TP53 and codons 12, 13, and 61 of K-ras were examined using direct sequencing. Results: TP53 mutations were observed in $10(33.3 \%)$ of patients, but K-ras mutations were absent. Nine (90\%) TP53 mutations were point mutations ( 7 missense and 2 silent mutations), and the most frequent substitution was a G:C to A:T transition. G:C to A:T transitions at the $\mathrm{CpG}$ site or $\mathrm{G}: \mathrm{C}$ to $\mathrm{T}: \mathrm{A}$ transversions were found in one patient each. No significant differences were found in the frequency of TP53 and K-ras mutations among patients in the 5 countries. Conclusions: Our findings suggest that endogenous mechanisms and exogenous carcinogens may affect the carcinogenic process in Peruvian gallbladder cancer patients, similar to that in Bolivian patients. Further studies with a larger sample size are needed to clarify these findings.
\end{abstract}

Keywords: Gallbladder cancer - mutation - tumor suppressor gene - proto-oncogene - Peru

Asian Pac J Cancer Prev, 20 (1), 289-294

\section{Introduction}

The age-standardized incidence rate of gallbladder cancer in Trujillo, Peru was 6.9 per 100,000 women and 2.3 per 100,000 men from 1988 through 1992, which ranked third in the world (Lazcano-Ponce et al., 2001). Peru has a high gallbladder cancer incidence even now, as shown by recent data from GLOBOCAN 2012, in which the age-standardized incidence rate was 5.5 per 100,000 women, ranking fifth worldwide, and 2.4 per 100,000 men (International Agency for Research on Cancer, GLOBOCAN 2012. Nevertheless, environmental and genetic factors underlying the pathogenic mechanism of gallbladder cancer have not elucidated. Specific factors associated with the development of gallbladder cancer in Peru are expected given the particularly high incidence of the cancer.

Tumor protein p53 (TP53) is the most frequently altered gene in human cancers (Freed-Pastor, 2012), and nucleotide substitution patterns, the presence of DNA strand bias, and carcinogenic factors associated with TP53 have been reported (Greenblatt et al., 1994). G:C to A:T transition mutations at the $\mathrm{CpG}$ site have been implicated in endogenous mechanisms of tumor development, and G:C to T:A transversions have been strongly associated with exposure to exogenous carcinogens (Pfeifer, 2000). The frequency of TP53 mutations in gallbladder cancer has been established as $31 \%$ to $71 \%$ (Takagi et al., 1994; Itoi et al., 1997; Moreno et al., 2005).

$\mathrm{K}$-ras is a proto-oncogene that exerts control in the mechanisms of cell growth and differentiation (Arrington et al., 2012). It is converted to an active oncogene through point mutations concentrated in codons 12,13 , and 61 by some carcinogens (Stolze et al., 2015). The frequency of K-ras mutations in gallbladder cancer patients was reported to be $0 \%$ to $41 \%$ (Kim et al., 2001; Deshpande et

${ }^{1}$ Instituto Nacional de Enfermedades Neoplásicas, Lima, Peru, ${ }^{2}$ Division of Preventive Medicine, ${ }^{4}$ Division of Molecular and Diagnostic Pathology, Niigata University Graduate School of Medical and Dental Sciences, ${ }^{3}$ Department of Clinical Engineering and Medical Technology, Niigata University of Health and Welfare, Niigata, ${ }^{5}$ Faculty of Health and Medical Sciences, Hokuriku University, Kanazawa, Ishikawa, Japan.*For Correspondence: troof441@gmail.com 
al., 2011; Kazmi et al., 2013). However, to our knowledge, data on the associations between these mutations and gallbladder cancer in Peru are limited. If causative factors for gallbladder cancer in Peruvians could be identified, it would be possible to investigate means of interfering with its development. Therefore, as a first step to determine causative factors of gallbladder cancer in Peruvians, we examined the frequency of TP53 and K-ras mutations in Peruvian gallbladder cancer patients, and compared this with our previous data obtained from Bolivian (Asai et al., 2014), Hungarian (Nagahashi et al., 2008), Chilean and Japanese (Yokoyama et al., 1998) patients.

\section{Materials and Methods}

\section{Subjects and gallbladder tissue}

A total of 30 patients ( 9 men and 21 women) with a diagnosis of gallbladder cancer by histopathological analysis at the Instituto Nacional de Enfermedades Neoplásicas (INEN, Lima, Peru) from September 1999 through January 2015 participated in this study. Gallbladder tissue was collected from each patient during surgery, fixed in formalin and embedded in paraffin wax, then used for histopathological diagnosis. Informed consent was obtained from all patients, and the study protocol was approved by the ethics committees of the INEN and Niigata University of Health and Welfare.

\section{DNA extraction}

Genomic DNA was extracted from formalin-fixed paraffin-embedded (FFPE) tissue using microdissection. A 3- $\mu \mathrm{m}$ section and adjacent serial $10-\mu \mathrm{m}$ sections were cut from the FFPE tissue and mounted onto glass slides. After deparaffinization in xylene 3 times for $10 \mathrm{~min}$ each, slides were washed 3 times in ethanol for $7 \mathrm{~min}$ each. Sections were stained with hematoxylin and eosin, and coverslipped for microscopic identification of the cancer site. Gallbladder cancer cells were scraped from non-coverslipped slides of $10-\mu \mathrm{m}$ sections. DNA was extracted from these cells using a DNA Isolator PS Kit (Wako Pure Chemical Industries, Osaka, Japan) according to operating procedures in the kit.

\section{Detection of TP53 mutations}

Mutations in exons 5 to 8 of the TP53 gene were detected using a nested PCR assay and direct sequencing of PCR products with previously described primers and procedures (Asai et al., 2014).

\section{Detection of K-ras mutations}

$\mathrm{K}$-ras mutations in codons 12,13 and 61 were detected using a nested PCR assay, PCR restriction fragment length polymorphism, and direct sequencing of PCR products. The analysis was performed using our previously described primers and procedures (Asai et al., 2014).

\section{Statistical analysis}

Differences in the frequency of TP53 and K-ras mutations were analyzed by a contingency table chi-square test. All statistical evaluations were performed on a personal computer using Stata 14 software (Stata Corp
LLC, College Station, TX, USA). $\mathrm{p}$ values of less than 0.05 (two-tailed) were regarded as statistically significant.

\section{Results}

Table 1 shows patient characteristics and TP53 mutation data. Mean ages were 66.1 years (standard deviation [SD], 5.8; range, 56-74 years) in male patients and 68.2 years (SD, 13.4; range, 33-88 years) in female patients, which was not significantly different $(p=0.66)$. The proportion of female patients was $70 \%$ $(21 / 30)$. A total of 29 patients $(96.7 \%)$ had a diagnosis of adenocarcinoma including mucinous adenocarcinoma, papillary adenocarcinoma, tubular adenocarcinoma, and signet ring cell carcinoma. One patient had a diagnosis of undifferentiated carcinoma. No patient had a diagnosis of anomalous union of the pancreaticobiliary duct (AUPBD). TP53 mutations were detected in 10 (33.3\%) of 30 patients, but no K-ras mutations were detected. The 10 mutations were distributed within a 360 bp range, from codon 180 through codon 299. Mutations at codons 190 and 281 were found in 2 patients each, and hotspot mutations at codons 245 and 248 were found in one patient each.

Table 2 shows the frequency of TP53 mutations in exons 5 to 8 . Of the 10 mutations, 4 (40\%) were found in exon 7 , which was the highest detection rate among the 4 exons. However no significant difference was found in the rate of mutation to codon number among the 4 exons $(\mathrm{p}=0.28)$.

Table 3 shows the types of TP53 mutations and their nucleotide alteration patterns. The 10 mutations were classified into 2 types: point mutations $(n=9,90 \%)$ and frameshift mutations $(\mathrm{n}=1,10 \%)$. Of the 9 point mutations, $7(70 \%)$ were missense mutations and 2 (20\%) were silent mutations. A frameshift mutation in which thymine was inserted at codon 299 was detected in one patient. The frequency of mutations by nucleotide alteration patterns was as follows: 6 transitions $(60 \%), 3$ transversions $(30 \%)$, and one insertion $(10 \%)$. The most frequently detected mutation was the $\mathrm{G}: \mathrm{C}$ to $\mathrm{A}: \mathrm{T}$ transition type, of which one was at the $\mathrm{CpG}$ site $(10 \%)$. The $\mathrm{G}: \mathrm{C}$ to $\mathrm{T}$ :A transversion mutation was detected in one patient (10\%).

Table 4 shows the frequency of TP53 and K-ras mutations among patients in countries with a high gallbladder cancer incidence. The frequency of TP53 mutations in Peruvian gallbladder cancer patients was $33.3 \%(10 / 30)$, which was the same as in Hungarian patients, and was the lowest value among the 5 countries. However no significant differences were found in the frequency of TP53 mutations among patients in the 5 countries $(p=0.41)$. Additionally, the frequency of transitions, transversions, and frameshift mutations in TP53 showed no significant differences among patients in the 5 countries. K-ras mutations were found in one Bolivian patient and one Hungarian patient only, so no significant difference was found in the frequency of this mutation among patients in the 5 countries. The TP53 mutation spectrum in Peruvian gallbladder cancer patients was similar to those seen in gallbladder cancer patients from Bolivia, which neighbors Peru. 
DOI:10.31557/APJCP.2019.20.1.289

Genetic Alterations in Peruvian Gallbladder Cancer Patients

Table 1. Patient Characteristics and Analytical Data of TP53 Mutations

\begin{tabular}{|c|c|c|c|c|c|c|c|}
\hline \multirow[t]{2}{*}{ Patient no. } & \multirow[t]{2}{*}{ Age (years) } & \multirow[t]{2}{*}{ Sex } & \multirow{2}{*}{$\begin{array}{l}\text { Cytologic } \\
\text { diagnosis }\end{array}$} & \multicolumn{2}{|c|}{ Mutation position } & \multirow{2}{*}{$\begin{array}{l}\text { Nucleotide } \\
\text { alteration }\end{array}$} & \multirow{2}{*}{$\begin{array}{l}\text { Amino acid } \\
\text { substitution }\end{array}$} \\
\hline & & & & Exon & Codon & & \\
\hline 1 & 80 & $\mathrm{~F}$ & muc & 8 & 281 & $\mathrm{GAC}$ to $\mathrm{CAC}$ & Asp to His \\
\hline 2 & 33 & $\mathrm{~F}$ & $\mathrm{AD}$ & - & - & - & - \\
\hline 3 & 72 & $\mathrm{~F}$ & pap & 6 & 190 & CCT to CTT & Pro to Leu \\
\hline 4 & 80 & $\mathrm{~F}$ & tub & - & - & - & - \\
\hline 5 & 63 & $\mathrm{~F}$ & tub & 6 & 190 & CCT to CTT & Pro to Leu \\
\hline 6 & 74 & M & $\mathrm{AD}$ & - & - & - & - \\
\hline 7 & 63 & M & $\mathrm{AD}$ & - & - & - & - \\
\hline 8 & 72 & $\mathrm{~F}$ & $\mathrm{AD}$ & 8 & 267 & CGG to $\mathrm{CAG}$ & Arg to Gln \\
\hline 9 & 60 & M & tub & - & - & - & - \\
\hline 10 & 65 & M & tub & - & - & - & - \\
\hline 11 & 78 & $\mathrm{~F}$ & pap & - & - & - & - \\
\hline 12 & 66 & $\mathrm{~F}$ & pap & - & - & - & - \\
\hline 13 & 71 & M & sig & 7 & 299 & $\mathrm{~T}$ insertion & Frameshift \\
\hline 14 & 65 & $\mathrm{~F}$ & $\mathrm{AD}$ & 8 & 281 & GAC to CAC & Asp to His \\
\hline 15 & 47 & $\mathrm{~F}$ & pap & - & - & - & - \\
\hline 16 & 50 & $\mathrm{~F}$ & $\mathrm{AD}$ & - & - & - & - \\
\hline 17 & 59 & $\mathrm{~F}$ & tub & - & - & - & - \\
\hline 18 & 67 & M & tub & 5 & 180 & GAG to GAA & Silent \\
\hline 19 & 70 & $\mathrm{~F}$ & UD & - & - & - & - \\
\hline 20 & 56 & M & tub & - & - & - & - \\
\hline 21 & 74 & $\mathrm{~F}$ & $\mathrm{AD}$ & - & - & - & - \\
\hline 22 & 57 & $\mathrm{~F}$ & tub & 7 & 237 & ATG to GTG & Met to Val \\
\hline 23 & 83 & $\mathrm{~F}$ & tub & - & - & - & - \\
\hline 24 & 65 & $\mathrm{~F}$ & tub & - & - & - & - \\
\hline 25 & 88 & $\mathrm{~F}$ & tub & - & - & - & - \\
\hline 26 & 72 & M & tub & - & - & - & - \\
\hline 27 & 70 & $\mathrm{~F}$ & tub & 7 & 248 & CGG to AGG & Silent \\
\hline 28 & 81 & $\mathrm{~F}$ & pap & 7 & 245 & $\mathrm{GGC}$ to $\mathrm{GAC}$ & Gly to Asp \\
\hline 29 & 79 & $\mathrm{~F}$ & pap & - & - & - & - \\
\hline 30 & 67 & M & $\mathrm{AD}$ & - & - & - & - \\
\hline
\end{tabular}

F, female; M, male; $\mathrm{AD}$, adenocarcinoma; muc, mucinous carcinoma; pap, papillary adenocarcinoma; tub, tubular adenocarcinoma; sig, signet ring cell carcinoma; UD, undifferentiated carcinoma; Asp, aspartic acid; His, histidine; Pro, proline; Leu, leucine; Arg, arginine; Gln, glutamine; Met, methionine; Val, valine; Gly, glycine

\section{Discussion}

This study demonstrated that one third of this cohort of Peruvian patients with gallbladder cancer had TP53 mutations, but none harbored K-ras mutation. The frequency of TP53 mutations in Peruvian patients was similar to that seen in studies of 36 patients from Bolivia (50.0\%), 18 from Hungary (33.3\%), 20 from Chile (55.0\%), and 22 from Japan (50.0\%) (Asai et al., 2014; Nagahashi et al., 2008; Yokoyama et al., 1998). The G:C to A:T transition mutation at the $\mathrm{CpG}$ site, which is implicated in endogenous mechanisms of tumor development, and the $\mathrm{G}: \mathrm{C}$ to $\mathrm{T}$ :A transversion mutation, implicated in associations with exogenous carcinogens, were detected in one patient each. These frequencies were similar to those seen in Bolivian patients, suggesting that gallbladder cancer development occurs through both
Table 2. Frequency of TP53 Mutations in Exons 5 to 8

\begin{tabular}{lccc}
\hline Exon & $\begin{array}{c}\text { No. of } \\
\text { codons }\end{array}$ & $\begin{array}{c}\text { No. of mutations } \\
\text { detected }\end{array}$ & $\begin{array}{c}\text { Mutations per } \\
\text { codon }\end{array}$ \\
\hline 5 & 61 & 1 & 0.02 \\
6 & 38 & 2 & 0.05 \\
7 & 37 & 4 & 0.11 \\
8 & 45 & 3 & 0.07 \\
P value & & & 0.28 \\
\hline
\end{tabular}

$P$ value was calculated using a 4 rows and 2 columns contingency table chi-square test.

endogenous and exogenous factors.

TP53 is a key gene for cancer development and its progression, and has the highest frequency of mutations in human cancers (Rivlinet et al., 2011). Thus, TP53 mutations are extensively investigated. The frequency of TP53 mutations was reported to be approximately 50\% 
Table 3. Type of TP53 Mutations and Pattern of Nucleotide Substitutions in Peruvian Patients with Gallbladder Cancer

\begin{tabular}{|c|c|c|c|c|c|c|c|c|c|c|c|}
\hline \multirow[t]{2}{*}{ Type of mutations } & \multicolumn{11}{|c|}{ Pattern of nucleotide alterations } \\
\hline & & & Transition & & & Transversion & & & Insertion & & \\
\hline Point mutations & 9 & $(90.0)$ & $\mathrm{G}: \mathrm{C}$ to $\mathrm{A}: \mathrm{T}$ & 5 & $(50.0)$ & $\mathrm{G}: \mathrm{C}$ to $\mathrm{T}: \mathrm{A}$ & 1 & $(10.0)$ & $\mathrm{T}$ insertion & 1 & $(10.0)$ \\
\hline Missense & 7 & $(70.0)$ & At $\mathrm{CpG}$ site & 1 & $(10.0)$ & $\mathrm{G}: \mathrm{C}$ to $\mathrm{C}: \mathrm{G}$ & 2 & $(20.0)$ & & & \\
\hline Silent & 2 & $(20.0)$ & At non-CpG site & 4 & $(40.0)$ & & & & & & \\
\hline Frameshift mutation & 1 & $(10.0)$ & A:T to $\mathrm{G}: \mathrm{C}$ & 1 & $(10.0)$ & & & & & & \\
\hline Total & 10 & & & 6 & $(60.0)$ & & 3 & $(30.0)$ & & 1 & $(10.0)$ \\
\hline
\end{tabular}

Numbers in parentheses are the percentage of the 10 mutations detected in this study.

Table 4. Frequency of TP53 and K-ras Mutations among Patients in Countries with a High Incidence of Gallbladder Cancer

\begin{tabular}{|c|c|c|c|c|c|c|c|c|c|c|c|}
\hline \multirow{3}{*}{ Sample no. } & \multicolumn{10}{|c|}{ Country } & \multirow[t]{2}{*}{$P$ value } \\
\hline & \multicolumn{2}{|c|}{ Peru } & \multicolumn{2}{|c|}{ Bolivia } & \multicolumn{2}{|c|}{ Hungary } & \multicolumn{2}{|c|}{ Chile } & \multicolumn{2}{|c|}{ Japan } & \\
\hline & 30 & & 36 & & 18 & & 20 & & 22 & & \\
\hline \multicolumn{12}{|l|}{ TP53 gene } \\
\hline No. of mutations & 10 & $(33.3)$ & 18 & $(50.0)$ & 6 & $(33.3)$ & 11 & $(55.0)$ & 11 & $(50.0)$ & 0.40 \\
\hline Transition type & 6 & $(20.0)$ & 10 & $(27.8)$ & 5 & $(27.8)$ & 11 & $(55.0)$ & 9 & (40.9) & 0.09 \\
\hline Transversion type & 3 & $(10.0)$ & 6 & $(16.7)$ & 1 & $(5.5)$ & 0 & $(0)$ & 4 & $(18.1)$ & 0.25 \\
\hline Frameshift type & 1 & $(3.3)$ & 2 & $(5.5)$ & 0 & $(0)$ & 0 & $(0)$ & 0 & $(0)$ & 0.53 \\
\hline \multicolumn{12}{|l|}{ K-ras gene } \\
\hline No. of mutations & 0 & (0) & & $(2.8)$ & 1 & ( 5.6$)$ & 0 & $(0)$ & 0 & $(0)$ & 0.51 \\
\hline
\end{tabular}

Numbers in parentheses are the percentage of mutations in each country; P values were calculated using a 2 rows and 5 columns contingency table chi-square test.

in human cancers, though the values vary according to the type of cancer (Soussi et al., 2000). The frequency of TP53 mutations in Peruvian gallbladder cancer patients in the present study (33\%) was within the reported ranges of other studies of gallbladder cancer, $31 \%$ to $71 \%$ (Takagi et al., 1994; Itoi et al., 1997; Moreno et al., 2005), as well as similar to our previous findings, $33 \%$ to $55 \%$ (Asai et al., 2014; Nagahashi et al., 2008; Yokoyama et al., 1998).

Of the 30 patients in this study, $29(96.7 \%)$ had a diagnosis of gallbladder adenocarcinoma, and the frequency of TP53 mutations was $34.5 \%$ (10/29). The frequency $(96.7 \%, 29 / 30)$ of the patients diagnosed as gallbladder adenocarcinoma was almost similar to that in Bolivian patients $(97.2 \%, 35 / 36)$ (Asai et al., 2014), Hungarian patients $(100 \%, 18 / 18)$ (Nagahashi et al., 2008), Chilean patients $(100 \%, 20 / 20)$ and Japanese patients $(100 \%, 22 / 22)$ (Yokoyama et al., 1998). In addition, the frequency (34.5\%) of TP53 mutations in the 29 adenocarcinoma patients of Peru was almost similar to TP53 overexpression positive rate $(29.2 \%, 21 / 72)$ in Chinese gallbladder adenocarcinoma patients using an immunohistochemistry method (Rashid et al., 2002). Judging by the results of this study, our data is thought of as reflect the trend of TP53 mutations in Peruvian patients with gallbladder cancer almost exactly.

Comparing the differences in the frequency of TP53 mutations among the 4 exons, one was for exon 5, 2 for exon 6,4 for exon 7, and 3 for exon 8 . No significant difference was found in frequency of mutations per codon number in each exon, so this results suggest that the mutations in exons 5 to 8 might be causing randomly.
However, the mutation in Peruvian patients differed from those in Hungarian and Chilean patients, namely, no mutation was found in exon 8 in Hungarian patients (Nagahashi et al., 2008), and a significant difference in frequency of mutations was found between exons $5(35 \%$, $7 / 20)$ and $8(5 \%, 1 / 20)$ in Chilean patients (Yokoyama et al., 1998).

The 10 mutations were detected between codons 180 and 299, and 2 were at hot spots (codons 245 and 248). No mutations were found at other hot spots, codons 135, 175, 176, 193, 273, and 306. Mutations at codons 190 and 291 were detected in each 2 patients, so these spots might be the specific hot spots for Peruvian patients with gallbladder cancer.

Food consumption contaminated with aflatoxin B1 was associated with an increased risk of hepatocellular carcinoma in previous studies (Kew, 2013). The presence of TP53 mutation at codon 249 in hepatocellular carcinoma patients has been reported to be associated with high risks of exposure to aflatoxins and hepatitis B virus (Ozturk, 1991). We have reported that high consumption of red chili pepper may be a risk factor for gallbladder cancer in Chilean and Hungarian people (Serra et al., 2002; Nakadaira et al., 2009). To clarify the association between red chili pepper consumption and gallbladder cancer, aflatoxins concentrations in red chili peppers of Chile, Bolivia, and Peru were measured using a HPLC system, the red chili peppers in these countries were contaminated with aflatoxins (Tsuchiya et al., 2011; Asai et al., 2012). These findings suggest that high consumption of red chili peppers contaminated with aflatoxins may be 
a risk factor for gallbladder cancer. In fact, one Hungarian patient with gallbladder cancer had a TP53 mutation at codon 249 (Nagahashi et al., 2008), though no mutation at this site was found in any Peruvian patients. Thus, additional studies with a large sample size are needed to clarify the association between the TP53 mutation at codon 249 and gallbladder cancer, especially in Chilean, Bolivian, and Peruvian patients.

Of the 10 mutations, 7 (70\%) were missense mutation which was emerged at the highest rate. In general, missense mutation altering amino acid sequence for protein synthesis has been accounted for approximately $75 \%$ of TP53 mutations (Freed-Pastor and Prives, 2012). The frequency of TP53 missense mutation obtained in this study was acceptable approximation $(70 \%)$. Frequency of frameshift deletion variant of the TP53 mutations in human cancers is known to be higher than that of the insertion variant (Hainaut et al., 1998), but that in Peruvian patient was the insertion variant. However, we were unable to clarify the reason why the insertion variant was detected in this study.

In examination regarding patterns of nucleotide alteration, $\mathrm{G}$ : $\mathrm{C}$ to $\mathrm{A}$ : $\mathrm{T}$ transition mutation was found in 5 $(50 \%)$ of the 10 patients, showing the highest frequency. Of the $5 \mathrm{G}: \mathrm{C}$ to $\mathrm{A}: \mathrm{T}$ transition mutations, mutation at the CpG site was detected in one patient (10\%). The patients with the mutations at this site are considered to have cancers developed through endogenous mechanisms or genetic factors (Ulrich et al., 2005). The reason why G:C to $\mathrm{A}: \mathrm{T}$ transition mutation at the $\mathrm{CpG}$ site is associated with endogenous carcinogenic are as follows: All cytosines methylated at the $\mathrm{CpG}$ site change to thymine after deamination spontaneously or by some mutagens easily (Tornaletti and Pfeifer, 1995). The G:C to A:T transition mutation at the $\mathrm{CpG}$ site was detected in only one patient. The frequency was low, but the development of gallbladder cancer in Peru may be associated with endogenous mechanisms. On the other hand, the presence of $\mathrm{G}: \mathrm{C}$ to $\mathrm{T}: \mathrm{A}$ transversion mutation of TP53 gene is considered to be cancer development by exogenous carcinogens (Hussain and Harris, 1999). Almost of the mutations are known to be induced by DNA damaging agents (exogenous carcinogens), or endogenous processes, or both (Povey, 2000). Although only one Peruvian patient had this type of mutation, the development of gallbladder cancer in Peru may be caused not only by endogenous mechanisms but also exogenous carcinogens as shown in Bolivian patients.

No significant difference was found in the frequency of TP53 mutations among the 5 countries' patients (range, $33.3 \%$ to $55.0 \% ; \mathrm{P}=0.58$ ). Also no significant differences were found in the frequency of transition, transversion, and frameshift mutations of TP53 gene among patients in the 5 countries. Our data shows that ethnic difference in the frequency of TP53 mutations is small. No K-ras mutation was found in Peruvian patients. Not only TP53 but also K-ras mutations are frequently found in human cancers (Riely and Ladany, 2008). This mutation plays an important role in the early stage of carcinogenesis of biliary mucosa, especially in association with AUPBD (Sagawa et al., 1998). Therefore, the frequency of K-ras
Genetic Alterations in Peruvian Gallbladder Cancer Patients

mutation has widely been reported to be $0 \%$ to $41 \%$ in gallbladder cancer patients according to the presence or absence of the AUPBD (Kim et al., 2001; Deshpande et al., 2011; Kazmi et al., 2013). A significant difference in the frequency of this mutation has been reported between gallbladder cancer patients with and without AUPBD in a previous study (Hanada et al., 1999). In this study, no patients had K-ras mutation because all patients had a diagnosis of gallbladder cancer without AUPBD. The frequency $(0 \%)$ of K-ras mutation in Peruvian patients was agreed with our previous reported value in Chile and Japan (Yokoyama et al., 1998). This result merely reflect the fact that we had no gallbladder cancer patients with AUPBD.

Our small sample size study could potentially have influenced our obtained frequency data. However, we used nearly the same number of the samples as our previous studies (Asai et al., 2014; Nagahashi et al., 2008; Yokoyama et al., 1998), and no significant difference was found in the frequency of TP53 and K-ras mutations among the 5 countries' patients. In addition, the frequency (10\%) of G:C to A:T transitions at the $\mathrm{CpG}$ site fell within the range $(0 \%$ to $33.3 \%)$ of the values observed in Bolivia, Hungary, Chile, and Japan. The frequency (10\%) of G:C to T:A transversions was also similar to the mean value $(3.6 \%, 2 / 55)$ observed in Bolivia, Hungary, Chile, and Japan. The frequency of these mutations was reported to be different according to the type of human cancer (Greenblatt et al., 1994): G:C to A:T transitions, 63\% for colon cancer, $20 \%$ for liver cancer; and G:C to T:A transversions, $40 \%$ for lung cancer, $6 \%$ for gastric cancer. Therefore, our data values may be considered reasonable for gallbladder cancer patients.

In summary, frequency of TP53 and K-ras mutations were $33.3 \%$ (10/30) and $0 \%$, respectively, in Peruvian patients with gallbladder cancer. No significant differences were found in the frequency of TP53 mutation, mutation types, and patterns of nucleotide alteration in Peruvian patients compared with those in the patients of countries with a high gallbladder cancer incidence. Especially, they were quite similar to those in the patients of Bolivia, located on the bordering country of Peru, suggesting effects of endogenous mechanisms and exogenous carcinogens in the carcinogenic process. However, an additional study with a large sample size will be needed to confirm our findings.

\section{Acknowledgments}

This work was supported by JSPS KAKENHI Grant Number JP24590767, and by a Grant-in-Aid for Advanced Research from Niigata University of Health and Welfare, 2015. We thank Sarah Williams, PhD, from Edanz Group (www.edanzediting.com) for editing a draft of this manuscript.

\section{References}

Arrington AK, Heinrich EL, Lee W, et al (2012). Prognostic and predictive roles of KRAS mutation in colorectal cancer. Int J Mol Sci, 13, 12153-68.

Asai T, Tsuchiya Y, Okano K, et al (2012). Aflatoxin 
contamination of red chili pepper from Bolivia and Peru, countries with high gallbladder cancer incidence rates. Asian Pac J Cancer Prev, 13, 5167-70.

Asai T, Loza E, Roig GV, et al (2014). High frequency of TP53 but not K-ras gene mutations in Bolivian patients with gallbladder cancer. Asian Pac J Cancer Prev, 15, 5449-54.

Deshpande V, Nduaguba A, Zimmerman SM, et al (2011). Mutational profiling reveals PIK3CA mutations in gallbladder carcinoma. BMC Cancer, 11, 60 .

Freed-Pastor WA (2012). Mutant p53: one name, many proteins. Genes Dev, 26, 1268-86.

Greenblatt MS, Bennett WP, Hollstein M, Harris CC (1994). Mutations in the p53 tumor suppressor gene: clues to cancer etiology and molecular pathogenesis. Cancer Res, 54, 4855-78.

Hainaut P, Hernandez T, Robinson A, et al (1998). IARC database of p53 gene mutations in human tumors and cell lines: updated compilation, revised formats and new visualisation tools. Nucleic Acids Res, 26, 205-13.

Hanada K, Tsuchida A, Iwao T, et al (1999). Gene mutations of $\mathrm{K}$-ras in gallbladder mucosae and gallbladder carcinoma with an anomalous junction of the pancreaticobiliary duct. Am J Gastroenterol, 94, 1638-42.

Hussain SP, Harris CC (1999). p53 mutation spectrum and load: the generation of hypotheses linking the exposure of endogenous or exogenous carcinogens to human cancer. Mutat Res, 428, 23-32.

International Agency for Research on Cancer. GLOBOCAN 2012: Estimated cancer incidence, mortality and preventive worldwide in 2012.

http://globocan.iarc.fr/Default.aspx. Accessed July 25, 2018.

Itoi T, Watanabe H, Yoshida M, et al (1997). Correlation of p53 protein expression with gene mutation in gall-bladder carcinomas. Pathol Int, 47, 525-30.

Kazmi HR, Chandra A, Nigam J, et al (2013). Prognostic significance of K-ras codon 12 mutation in patients with resected gallbladder cancer. Dig Surg, 30, 233-9.

Kew MC (2013). Aflatoxins as a cause of hepatocellular carcinoma. J Gastrointestin Liver Dis, 22, 305-10.

Kim YT, Kim J, Jang YH, et al (2001). Genetic alterations in gallbladder adenoma, dysplasia and carcinoma. Cancer Lett, 169, 59-68.

Lazcano-Ponce EC, Miquel JF, Muñoz N, et al (2001). Epidemiology and molecular pathology of gallbladder cancer. CA Cancer J Clin, 51, 349-64.

Moreno M, Pimentel F, Gazdar AF, Wistuba II, Miquel JF (2005). TP53 abnormalities are frequent and early events in the sequential pathogenesis of gallbladder carcinoma. Ann Hepatol, 4, 192-9.

Nagahashi M, Ajioka Y, Lang I, et al (2008). Genetic changes of p53, K-ras, and microsatellite instability in gallbladder carcinoma in high-incidence areas of Japan and Hungary. World J Gastroenterol, 14, 70-5.

Nakadaira H, Lang I, Szentirmay Z, et al (2009). A case-control study of gallbladder cancer in Hungary. Asian Pac J Cancer Prev, 10, 833-6.

Ozturk M (1991). p53 mutation in hepatocellular carcinoma after aflatoxin exposure. Lancet, 338, 1356-9.

Pfeifer GP (2000). p53 mutational spectra and the role of methylated CpG sequences. Mutat Res, 450, 155-66.

Povey AC (2000). DNA adducts: endogenous and induced. Toxicol Pathol, 28, 405-14.

Rashid A, Ueki T, Gao YT, et al (2002). K-ras mutation, p53 overexpression, and microsatellite instability in biliary tract cancers: A population-based study in China. Clin Cancer Res, 8, 3156-63.

Riely GJ, Ladany M (2008). Kras mutations. J Mol Diagn, 10,
493-5.

Rivlin N, Brosh R, Oren M, Rotter V (2011). Mutations in the p53 tumor suppressor gene: Important milestones at the various steps of tumorigenesis. Genes Cancer, 2, 466-74.

Sagawa M, Saito Y, Fujimura S, Linnoila RI (1998). K-ras point mutation occurs in the early stage of carcinogenesis in lung cancer. Br J Cancer, 77, 720-3.

Serra I, Yamamoto M, Calvo A, et al (2002). Association of chili pepper consumption, low socioeconomic status and longstanding gallstones with gallbladder cancer in a Chilean population. Int J Cancer, 102, 407-11.

Soussi T, Dehouche K, Béroud C (2000). p53 website and analysis of $\mathrm{p} 53$ gene mutations in human cancer: forging a link between epidemiology and carcinogenesis. Hum Mutat, 15, 105-13.

Stolze B, Reinhart S, Bulllinger L, et al (2015). Comparative analysis of KRAS codon 12, 13, 18, 61, and 117 mutations using human MCF10A isogenic cell lines. Sci Rep, 5, 8535.

Takagi S, Naito E, Yamanouchi H, et al (1994). Mutation of the p53 gene in gallbladder cancer. Tohoku J Exp Med, 172, 283-9.

Tornaletti S, Pfeifer GP (1995). Complete and tissue-independent methylation of $\mathrm{CpG}$ sites in the $\mathrm{p} 53$ gene: implications for mutations in human cancers. Oncogene, 10, 1493-9.

Tsuchiya Y, Terao M, Okano K, et al (2011). Mutagenicity and mutagens of the red chili pepper as gallbladder cancer risk factor in Chilean women. Asian Pac J Cancer Prev, 12, 471-6.

Ulrich CM, Curtin K, Samowitz W, et al (2005). MTHFR variants reduce the risk of $\mathrm{G}: \mathrm{C}->\mathrm{A}: \mathrm{T}$ transition mutations within the p53 tumor suppressor gene in colon tumors. J Nutr, 135, 2462-7.

Yokoyama N, Hitomi J, Watanabe H, et al (1998). Mutations of p53 in gallbladder carcinomas in high-incidence areas of Japan and Chile. Cancer Epidemiol Biomarkers Prev, 7, 297-301.

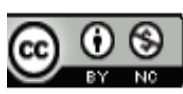

This work is licensed under a Creative Commons AttributionNon Commercial 4.0 International License. 